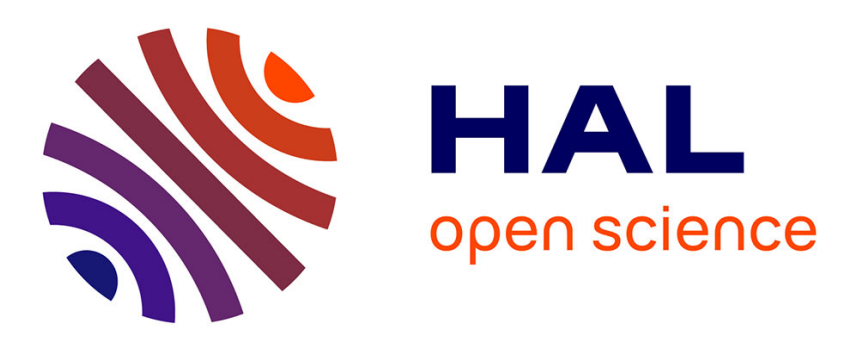

\title{
Imagerie médicale et art contemporain, rencontres autour d'un corps virtuel \\ Rémy Potier
}

\section{To cite this version:}

Rémy Potier. Imagerie médicale et art contemporain, rencontres autour d'un corps virtuel. Recherches en psychanalyse, 2011, Psychanalyse, corps et Société / Psychoanalysis, the Body and Society, 2 (12), pp.130-139. 10.3917/rep.012.0130 . hal-01516342

\section{HAL Id: hal-01516342 \\ https://hal.science/hal-01516342}

Submitted on 13 Jul 2017

HAL is a multi-disciplinary open access archive for the deposit and dissemination of scientific research documents, whether they are published or not. The documents may come from teaching and research institutions in France or abroad, or from public or private research centers.
L'archive ouverte pluridisciplinaire HAL, est destinée au dépôt et à la diffusion de documents scientifiques de niveau recherche, publiés ou non, émanant des établissements d'enseignement et de recherche français ou étrangers, des laboratoires publics ou privés. 


\section{Recherches en Psychanalyse - Research in Psychoanalysis}

12 |2011- Psychanalyse, corps et Société

Psychoanalysis, the Body and Society

Dossier "Psychanalyse, corps et société"

Imagerie médicale et art contemporain, rencontres autour d'un corps virtuel

Medical Imaging and Modern Art: Encounters over a Virtual Body

Rémy Potier

\section{Résumé :}

L'art contemporain offre un regard singulier et révélateur concernant le corps, tel qu'il est aujourd'hui vécu. À partir de cet intérêt porté au corps, la question de la virtualisation de celui-ci est également devenue source d'inspiration. Des artistes se saisissent ainsi d'images véhiculées par la médecine à travers l'imagerie pour mettre en scène ce corps virtuel. C'est cette actualisation du corps virtuel qui interpelle les artistes. Ils intègrent ces images du corps issues de la médecine dans leur dispositif artistique. Cette rencontre entre art et médecine à l'heure des appareillages technique engage la question de la place acquise par ces images dans notre culture, et la façon dont celles-ci illustrent ce que devient notre rapport à l'altérité puis à la mort. L'une des illustrations du corps, aujourd'hui, dans sa visibilité la plus intime, est portée par l'imagerie médicale. Cette technique est le média incontournable par lequel nous nous représentons notre corps de chair, sujet à la maladie et voué à la mort. Il convient de se laisser saisir par l'art contemporain à partir de ce qu'il donne à penser de la rencontre du sujet avec l'imagerie médicale. Le malaise dans la culture, est aujourd'hui étroitement lié à ces immersions dans l'image (virtuelle) dont l'accès et les pratiques sont de plus en plus répandues, au-delà même du domaine de la santé. C'est ainsi que l'art depuis le XXe siècle montre du corps ce que les techniques de visualisation ont permis de voir les unes après les autres, s'insinuant dans la culture, au point de modifier le rapport au corps et à ses images. Les rayons $X$, les photographies en gros plan, la macrophotographie vont être enrôlés au service de l'art. Ce détour par l'art est très instructif, il permet de montrer combien le regard porté sur l'image médicale déroute et met à l'épreuve l'idée même de représentation.

\section{Abstract :}

Modern art offers us a singular and revealing perspective on the body and the way it is experienced today. Based on this interest in the body, the latter's virtualization has also become a source of artistic inspiration. To present this virtual body, artists sometimes use images produced by medical imaging technologies. The actualization of the virtual body poses a question for them. The images of the body produced by medicine are thus integrated into their artistic works. In an era of sophisticated technological equipment, this encounter between art and medicine raises the question of the position of these images in our culture and the way in which they illustrate our relationship to otherness and to death. Today, medical imaging provides one of the key illustrations of the body, seen at its most intimate. This technology is the inescapable medium through which we imagine our living body, a body that is subject to illness and doomed to death. It is therefore useful to look at contemporary art and what it tells us about the subject's encounter with medical imaging. The cultural discontent of today is clos $\$$ 
related to our immersion in the (virtual) image, while the access to and use of these technologies is becoming 
increasingly widespread and extends far beyond the field of healthcare. Since the 20th century, art has been trying to show the aspects of the body that these techniques have allowed us to see, as they are gradually seeping into our culture one after another, and ultimately modify our relationship to the body and its representation. Xrays, close-ups, macrophotography-each of these have been enlisted in the service of art. This detour through art can teach us a great deal, helping us see the extent to which looking at these images confounds and challenges the very idea of representation.

Mots-clefs : psychanalyse, imagerie médicale, art contemporain, culture

Keywords : psychoanalysis, medical imaging, contemporary art, culture

\section{Plan :}

La place de la médecine aujourd'hui

De l'imagerie à l'art, le dévoilement du corps

Discussion

Illustrations

Discussion 2

Conclusion

L'art contemporain offre un regard singulier et révélateur concernant le corps, tel qu'il est conçu et vécu aujourd'hui. Á partir de cet intérêt porté au corps, la question de sa virtualisation est devenue source d'inspiration. Ainsi des artistes se saisissent d'images véhiculées par la médecine pour mettre en scène ce corps virtuel. Cette actualisation du corps virtuel au niveau de la santé interpelle les artistes. Ils intègrent ces images du corps issues de la médecine dans leur dispositif artistique. Cette rencontre entre art et médecine - à I'heure des appareillages technique - pose la question de la place acquise par ces images dans notre culture. Elles illustrent ce que devient notre rapport à notre corps propre, mais aussi à l'altérité puis à la mort. L'une des illustrations du corps aujourd'hui, dans sa visibilité la plus intime, est portée par l'imagerie médicale. Cette technique est le média incontournable par lequel nous nous représentons notre corps de chair, sujet à la maladie et voué à la mort. Le malaise dans la culture, est aujourd'hui étroitement lié à ces immersions dans l'image (virtuelle) dont l'accès et les pratiques sont de plus en plus répandues. L'art depuis le XXe siècle montre du corps ce que les techniques de visualisation ont permis de voir les unes après les autres. Les artistes interrogent la manière dont ces techniques modifient, dans la culture, le rapport au corps et à ses images. Des exploits marginaux, comme le travail d'Orlan, deviennent des modalités courantes de la biotechnologie contemporaine qui a l'ambition de rectifier la forme organique en intervenant de plus en plus tôt, notamment sur les gènes. Cette transformation du corps est motivée par la recherche d'un corps parfait et idéal. Quelles que soient les finalités, thérapeutiques, esthétiques ou ludiques, ces transformations corporelles poursuivent un corps idéal, un corps de rêve qui, par l'intervention médicale, doit devenir corps réel. L'art contemporain permet de penser l'imagerie médicale dans ce contexte.

Les dispositifs que proposent certains artistes à partir de l'imagerie médicale, révèlent l'aspect refoulé du corps contemporain, sa dimension sexuelle, éradiquée dans la méthodologie médicale.

Il s'agit d'abord de s'interroger sur la place dont la médecine jouit dans le social, évaluer son rôle quant à la représentation du corps. L'envers du dispositif médical se trouve illustré par les œuvres de certains artistes, poursuivant la question du corps. Le dispositif proposé par 
Mona Hatoum, Foreign Body, permettra d'illustrer et d'interroger l'effet des images médicales, sa résonance intime. Cet attrait des images médicales pourra s'illustrer de façon différente chez d'autres artistes. Je souhaite dégager depuis la psychanalyse la dimension inconsciente de ce corps à corps avec la technique. II s'agit d'accompagner ce processus par lequel la technique change l'homme et l'image de lui-même.

\section{La place de la médecine aujourd'hui}

La médecine, aujourd'hui, est technoscientifique. Je voudrais montrer que ce fait induit une nouvelle représentation du corps dans le social, qui engage le rapport du sujet à l'image de son corps. Nous pouvons repérer un processus de normalisation au niveau de la santé mais aussi au niveau anthropologique. La mise en évidence de cette dimension invite à révéler l'enjeu d'une réflexion interdisciplinaire à propos de la médecine et plus spécifiquement à propos de l'imagerie. L'image du corps en médecine est celle du corps interne, un corps construit, un corps autre, qui nous est et nous demeure étranger. Cette image, nous la devons par ailleurs aux techniques de virtualisation et à la puissance de calcul des ordinateurs. L'image en médecine relève d'un environnement virtuel dans la médiation intelligible du modèle qui constitue l'image. Le sujet est confronté à une expérience d'immersion. L'image est celle du corps, mais ce corps est pour la médecine et seulement lisible par elle. Ces spécificités se retrouvent dans le social à deux niveaux. Celui du vocabulaire concernant le corps, imprégné du vocable médical. L'autre niveau est celui de la question du "transfert ", au sens psychanalytique, tel que le rencontre les médecins aujourd'hui.

Le langage de la médecine, ses mots, ne sont pas sans effet sur la construction de la représentation du corps aujourd'hui. Le scanner, l'échographie, l'IRM, ont désormais leur place à côté de la radiographie conventionnelle dans le parcours de santé de l'individu aujourd'hui.
"Mon scanner n'a pas inquiété mon médecin ", " mon écho était bizarre ", depuis ces paroles de patients, je voudrais souligner l'implication des techniques d'imagerie médicale dans le vocabulaire commun contemporain. Cette évolution dans la langue doit étonner, au sens où il s'agit d'entendre ce que l'Inconscient a pu y former à partir d'expériences personnelles vécues dans un corps à corps avec ces techniques, le plus souvent à partir d'une ignorance totale du fonctionnement et des implications de celles-ci. Dans ces paroles, le corps est désigné par ce qui le révèle, l'organe laisse la place à la technique d'investigation, et l'expérience vécue, condensée dans une formule magique relevant du high tech. On ne saurait plus aujourd'hui parler de notre corps et de son fonctionnement sans recourir au vocabulaire médical. Le corps est pour nous un ensemble d'organes sièges de processus physiologiques et biochimiques. Ainsi, nous désignons et localisons nos maladies selon une géographie et une terminologie de type médical, même si celles-ci ne recoupent pas forcément la nomenclature officielle.

Le savoir médical nourrit bien la langue et les représentations mais reste hermétique aux patients. Ces emprunts de mots, il est vrai, orientent notre représentation et notre expérience du corps, et le vocabulaire technique utilisé permet de faire de notre corps un objet extérieur avec lequel il est possible de prendre un minimum de distance, voire de conjurer les inquiétudes qu'il nous inspire. Mais, depuis ce qui s'organise, un reste fait retour. C'est ce reste qui intéresse l'investigation psychanalytique. Le rapport au corps comme pur objet extérieur est devenu pour les médecins une évidence. La spécificité du savoir médical contemporain, à partir du vocabulaire technique que nous lui empruntons, consiste en ce que ce corps reconstruit s'impose à nous comme un objet supposé, qui est en réalité une construction, avec lequel il convient - et ce n'est pas toujours possible - de prendre un minimum de distance. Mais, cet objet corps n'épuise pas, pour le malade, la vérité de la maladie. 
C'est dans ce contexte que s'adressent les demandes des patients et que se joue leur transfert $^{1}$ au corps médical. Or la religiosité, aujourd'hui, se situe au niveau des prouesses technologiques. La société encouragée par les médias, émerveillée et fascinée, pense que ce " progrès médical " est sans limite. Les séries télévisées, comme Urgences, Grey's anatomy ou Docteur House, deviennent la vitrine des dernières nouveautés des technologies médicales et les scénarii rédigés par des médecins promeuvent le vocable médical. L'incompréhension du spectateur stimule l'identification héroïque au staff médical. Le pouvoir sur le corps étourdit et fait écho aux pratiques devenues courantes, engageant les actions sur soi. Alors que la science est progrès, la médecine fait des miracles, ce message constitue sa religiosité... Chaque avancée semble effacer les traces de la précédente mais, pourtant, le progrès en médecine se construit, en fait, sur un arrière-fond hanté par l'angoisse de la mort, laquelle se veut mise à l'écart et est laissée sans voix. Ainsi plusieurs visions contradictoires s'affrontent. Le regard que porte le médecin sur sa science n'est jamais celui du patient sur sa maladie et les mots qu'il apprend des médias accentuent les difficultés de frontières et rendent difficile la rencontre.

Ce transfert du réel du corps sur la virtualité des normes paramétriques des images, des chiffres, véritable nouvelle réalité, aspire vers l'extérieur la conscience du corps. La médecine technoscientifique superpose une science médicale à la subjectivité, pouvant au mieux ou au pire tenir lieu de nouvelle identité du corps. Les avancées de la psychopharmacologie, les interférences électriques, cerveau-ordinateur comme simple accroissement des potentialités, ne font plus la différence entre son propre corps, les neuromédiateurs et les organes externes. Les médecins constatent déjà que des patients disent aller mal parce que la médecine dit qu'il y a un risque d'être malade ou parce qu'elle ne sait pas trouver pourquoi il va mal ; au contraire, un patient peut aller bien si la médecine lui donne quitus de son état actuel.
Nous ne sommes plus capables de discerner quelle est la vérité de notre corps.

Cette perte de confiance dans les signaux de son corps à l'échelon individuel a sa traduction amplifiée à l'échelon collectif. La vérité du corps est du côté du médecin.

Ainsi, le progrès médical fonde un nouveau réel toujours plus efficace, sans penser que la médecine change l'homme.

Mon propos va s'attacher à révéler ce qui se produit au coeur de cette rencontre avec l'imagerie $d u$ corps interne, effet que permettent de faire sentir, de façon spécifique les artistes qui utilisent l'imagerie dans leur dispositif artistique.

\section{De l'imagerie à l'art, le dévoilement du corps}

Il est utile de se laisser saisir par l'art contemporain, par ce qu'il donne à penser de la rencontre du sujet avec l'imagerie médicale. Je propose tout d'abord de présenter une œuvre de Mona Hatoum. II s'avère que beaucoup d'artistes contemporains se sont inspirés des technologies médicales. Dès la dernière décennie du XXe siècle, est apparue une nouvelle vague du Body Art. En guise d'exemple, l'œuvre de Mona Hatoum intitulée Foreign Body (1994).

Il s'agit d'une installation vidéo qui montre des images endoscopiques de l'intérieur du corps de l'artiste. Mon propos consiste à relever la logique fantasmatique qui s'articule comme corrélat du discours et des dispositifs que produit la technique scientifique. Je souhaite montrer que l'artiste met en scène cette logique de façon à transmettre son regard propre sur l'objet. Le regard de l'artiste se déploie dans les dispositifs et traduit quelque chose du malaise du regard contemporain sur le corps.

L'œuvre de Mona Hatoum, se situe dans la tradition du Body Art. Le corps a toujours joué un rôle important dans l'œuvre de cette artiste. La question de la surveillance par les caméras est un autre thème récurrent dans son œuvre. En 1980, elle a réalisé Don't Smile, You're on Camera, une performance " live » où elle filmait 
le public. Ces images vidéo étaient mélangées avec d'autres images. Dans son dispositif, l'image d'une personne bien habillée se mélangeait avec celle d'une personne nue ou avec une image rayon- $X$.

Les images projetées sur l'écran donnaient l'impression que le regard de la caméra glissait sous les vêtements et sous la peau. L'artiste met en scène la problématique voyeuriste, de façon à placer les spectateurs dans le renversement qui va du voyeurisme à l'exhibitionniste. Elle parvient à produire une sorte de métonymie de l'effet qui saisit le sujet contemporain qui semble voué au monde de l'immersion dans l'image. Or ce voyeurisme se réalise dans l'œil de la caméra qui pénètre effectivement l'intérieur du corps dans Foreign Body (1994). Ce regard envahissant est à la fois quelque chose de fascinant et d'effrayant, voire de violent. Cette œuvre semble impliquer simultanément un étonnement vis-à-vis des parties inconnues du corps et une critique de la technique de l'appropriation par l'imagerie, plus encore, elle en restitue la part angoissante.

Foreign Body consiste en une sorte de cabine dans laquelle on doit entrer pour regarder et sentir l'œuvre.

Sur le sol de la cabine, il y a un écran rond sur lequel sont projetées des images agrandies de la surface et de l'intérieur du corps de l'artiste. ${ }^{2}$ On peut suivre la caméra endoscopique qui tantôt effleure la peau et tantôt pénètre les orifices, comme l'anus et le vagin, pour filmer l'intérieur du corps. Les mouvements de la caméra sont accompagnés par le bruit amplifié de la respiration et de la pulsation cardiaque de l'artiste : au moment où la caméra reste en dehors du corps, on entend le son de la respiration, dès qu'elle y entre, on entend le battement du cœur. De ce fait, le spectateur qui se trouve dans la cabine assez étroite, devant marcher sur l'écran, peut se sentir comme absorbé dans le corps de l'artiste. Il est pris dans un " cercle étrangement intime ", entre l'extériorité et l'intériorité du corps.

Restée consciente durant l'examen médical, Mona Hatoum a participé à la réalisation de cette vidéo qui dévoile au spectateur les régions les plus intimes de son corps, auxquelles seul le regard médical, organe de contrôle, a en principe accès. C'est en ce sens qu'agit tout d'abord le titre de l'œuvre, désignant la caméra, extension du regard scientifique, comme un élément extérieur pénétrant le corps du patient, se l'appropriant, puis en rapportant des images déconstruites. À tel point que le corps examiné, pourtant familier, devient lui aussi étranger, méconnaissable pour l'individu auquel il appartient - sans compter que l'imagerie médicale révèle parfois une anomalie que le sujet ne soupçonnait pas, renforçant encore le sentiment d'aliénation vis-à-vis de son propre corps. En outre, l'expression "Foreign Body " renvoie à la position du spectateur au sein du dispositif de l'installation. En effet, en s'engageant dans la structure où est installée la vidéo endoscopique, celui-ci s'immisce dans un corps inconnu, qu'il est invité à sonder à travers des images dont les proportions dépassent l'échelle réelle, amplifiant l'effet d'immersion dans un territoire autre, ici délimité par l'architecture circulaire. Que le spectateur contourne l'image en longeant la paroi interne de la structure qui encercle la vidéo, ou qu'il franchisse obliquement la surface de projection, il n'échappe pas à la confrontation avec ce corps révélé sous ses aspects biologiques.

Le type de structure, conçu par l'artiste pour accueillir la vidéo endoscopique, dicte la réception de l'œuvre par le spectateur : la forme relativement close du cylindre - seules deux portes étroites permettent d'y pénétrer suggère un espace privé, à l'accès réservé. Ce sentiment est renforcé par l'obscurité presque totale de l'environnement (les images vidéo sont l'unique source de lumière de l'installation). Puis, une fois la porte franchie, la nature extrêmement intime des images projetées inflige inévitablement au spectateur un statut de voyeur. Par ailleurs, Foreign Body renvoie à la notion de surveillance et à la corrélation entre regard et pouvoir, principe largement développé par Foucault. En effet, la rencontre, au sein d'un espace architectural, 
entre le corps de l'artiste et le regard du spectateur, fonctionne comme une mise en espace du pouvoir exercé par l'institution médicale, à travers le regard scientifique qu'elle porte sur le corps vulnérable du patient.

Le Foreign Body n'est pas le corps propre, ce n'est pas mon corps, mais le corps d'un autre. Mais, pour l'artiste, le corps montré sur l'écran n'est pas non plus tout à fait familier. L'intrusion de quelque chose d'étranger dans son corps renvoie à la caméra, par où Mona Hatoum signifie combien la médecine confronte à une expérience intrusive par excellence.

\section{Discussion}

Que dire de cette rencontre avec l'étrangeté des images ? L'écran du Foreign Body brise le miroir du narcissisme. Foreign Body bouleverse profondément la relation entre corps propre et corps étranger, mais cela ne veut pas dire que celui-là serait remplacé par celui-ci.

Jenny Slatman ${ }^{3}$, fait l'hypothèse, à partir d'une perspective phénoménologique, que les nouvelles images du corps interne, offertes par l'imagerie médicale (échographie, endoscopie, IRM, TDM et TEP), affectent l'image du corps en tant qu'identité corporelle vécue. Elle ajoute que " puisque ces nouvelles images - morcelées et presque méconnaissables - ne sont pas facilement intégrables dans l'image spéculaire de notre corps, il faut dépasser la théorie du narcissisme, qui réduit l'image du corps à une "image visuelle" ". ${ }^{4}$ Slatman propose alors une théorie de "l'image affective ". Je pense, pour ma part, que l'œuvre de Mona Hatoum, plutôt que de vouloir nous apprivoiser avec l'image du corps interne, témoigne davantage de l'effet engagé dans l'expérience visuelle comme angoisse de morcellement.

L'un des enjeux dégagés par Lacan avec le stade du miroir semble impliqué ici. L'enfant reconnaît son image spéculaire, mais il ne perçoit pas encore qu'il y a une différence entre cette image et soi-même: il n'y a qu'une " méconnaissance " (Lacan, 1949, 98). L'enfant ne reconnaît pas encore l'altérité de l'image, il se confond avec l'autre. Le dispositif que propose Mona Hatoum traduit cette étrange alternative en imposant les images du corps interne en interaction avec les mouvements effectués par le spectateur dans la cabine. Dans l'expérience de Hatoum, les sons et les images laissent sans voix; elles traduisent, depuis un dispositif spéculaire, une expérience préspéculaire (pré-visuelle). Aussi, mon idée est que la culture dans laquelle nous vivons nous offre continuellement une multiplicité d'images qui peuvent servir comme miroir dans lequel nous nous réfléchissons, et c'est ce malaise précis qu'entend faire passer Hatoum par son dispositif artistique. Au lieu d'affirmer l'image du corps en tant que corps propre, les images du corps interne montrent le corps étranger et absent, et ne peuvent donc pas fonctionner comme des images idéales. Pas d'identification possible.

Les images du corps interne appartiennent au " corps morcelé » qui précède l'unité imaginaire du stade du miroir. Et, dans la vie d'adulte, il peut y avoir une régression de ce corps morcelé sous la forme d'une "désintégration agressive de l'individu ", par exemple dans le cas de I'hystérie ou plus simplement à l'occasion de la maladie.

Selon Lacan, les images du corps morcelé restent en dehors du champ de la vison, elles sont exoscopiques. Elles restent en dehors du domaine de l'imaginaire et en dehors du domaine du symbolique; elles ne sont pas de l'ordre de la représentation. Puisque Foreign Body nous offre des images du "corps morcelé ", ces images endoscopiques sont bel et bien exoscopiques. La figuration du corps interne n'est pas à intégrer dans l'image du corps en tant qu'unité du corps. Ce corps morcelé se manifeste au moment où l'image du corps est brisée. Ce qui est en jeu ici est une présentation visuelle de ce qui ne peut être représenté, de ce qui ne peut être assimilé à l'image du corps : en cela réside l'étrangeté. C'est la raison pour laquelle Foreign Body peut être rencontré comme une figuration de ce que ces images du corps sont potentiellement. 


\section{Illustrations}

L'image médicale peut être considérée comme une expérience d'image inséparable de son énonciation. C'est une image en travail, une image-acte, un geste de production ajouté à l'acte de la réception et de sa contemplation. Dans le travail de Mona Hatoum, l'étrangeté est au cœur du message artistique, soit comme impossibilité de se référer à un corps unifié, soit comme un message de désintégration identitaire, qui fait signe vers l'impossible de l'identification.

L'œuvre de Mona Hatoum traite de l'expérience du déplacement, de la désorientation, de la reconstruction de l'identité, du désordre que génère la vie et $d u$ profond sentiment de malaise qui en découle.

Depuis la Seconde Guerre mondiale et la popularisation des images de rayons $X$, beaucoup d'artistes ont utilisé de telles images. Dans un premier temps, ce qui a guidé le travail de plusieurs d'entre eux a été la réflexion sur ce monde intérieur et transparent représenté par les images médicales. C'est le cas, par exemple, du travail de Francis Bacon, qui, dans les années 1950, s'est servi d'un livre de radiologie Positioning in radiology -- écrit en 1934 par Kathleen Clara Clark, l'utilisant comme une espèce de manuel pour la production de quelques-unes de ses œurres, telles Head Surrounded by sides of beef (1954), où l'artiste utilise les images de rayons $X$ comme références pour représenter un corps vivant avec la forme d'une carcasse.

Le corps malade est saisi en tant que tel, comme chez l'artiste américaine Laura Ferguson, connue pour sa série The visible skeleton. L'artiste commença à expérimenter la déformation physique à la suite d'une scoliose aiguë. À l'âge de treize ans, Laura Ferguson fut soumise à une chirurgie de fusion de l'épine dorsale. Les transformations dans son corps l'amenèrent à expérimenter de nouveaux concepts d'espace et de gravité, et à développer une nouvelle conscience de certains processus comme la respiration et le mouvement.
Initialement, Laura Ferguson reprend les images de ses registres médicaux et, ensuite, d'autres registres produits par des médecins, spécialement pour la réalisation de ses œuvres. Selon l'artiste, cette série raconte sa propre trajectoire et montre comment elle a transformé les expériences de son corps médicalisé en art.

Face à l'effet éprouvé par l'expérience visuelle à laquelle l'imagerie livre le corps, le sujet reconduit à une expérience pré-spéculaire est nécessairement étreint par l'angoisse, si bien que le mouvement artistique peut être pensé comme l'acte de tenter une expérience constituante de l'angoisse, d'apprendre à s'en servir pour se refaire un corps. En incorporant et en transformant des images produites par les technologies médicales (rayons $X$, tomographies, résonances), l'artiste suscite une nouvelle vision du corps et redéfinit, en outre, la notion de portrait en mettant en question les significations de l'image unifiée de soi.

Pour prendre un autre exemple, l'artiste brésilienne Monica Mansur explore les images de rayons $X$, d'endoscopie, d'ultrason, de tomographie et les fait interagir avec les interprétations culturelles. Son œuvre propose de mettre en scène les corps scientifiques médicalisés dans un scénario artistique. Pour Rosana Horio Monteiro, qui a travaillé sur son œuvre :

[...] la popularisation des images
médicales dans différents contextes
médiatiques (cinéma, télévision, publicité) a
fourni à un large public un regard qui était
précédemment réservé à l'œil spécialisé du
seul médecin. Elle a ainsi contribué à la
création d'une culture dépendant des
images et des technologies qui les
produisent. Les processus d'éclairage de
l'intérieur du corps humain et la
transparence deviennent ainsi, de plus en
plus, des produits ou des artefacts
culturels. ${ }^{5}$

Monica Mansur présente régulièrement l'exploration d'images médicales dans ses travaux de graphiste. Depuis 1995, année de la première exposition de ses œuvres produites à partir de 
rayons $X$ et imprimées sur sparadrap et gaze, jusqu'aux années plus récentes où elle a créé ce qu'elle appelle des " paysages cristaux " ou rephotographies, Monica Mansur explore les possibilités de création à travers la reproduction et réfléchit sur l'esthétique de la répétition.

Les rephotographies sont des images rephotographiées et imprimées à partir de plusieurs examens médicaux. L'artiste photographie en fait l'image résultant des examens, la numérise et la retravaille. Dans ce processus, elle enlève certaines parties, augmente ou diminue la lumière, et modifie l'espace physique. L'image est imprimée sur différents supports comme le sparadrap et la gaze. En 1996, elle se familiarise avec I'utilisation de la vidéo et crée une installation avec des images mobiles de l'intérieur de l'estomac et du côlon humains.

Les images utilisées par Monica Mansur peuvent être celles de son propre corps ou de n'importe quel autre : ce sont des non-identités. Rassemblées aléatoirement, ces images peuvent être celles de patients décédés, malades ou non. ${ }^{6}$

\section{Discussion 2}

Dans les travaux que je viens d'exposer, la question du corps et de notre identité est au cœur du message artistique, soit comme impossibilité de se référer à un corps unifié, soit comme un message de désintégration identitaire, qui fait signe vers l'impossible de l'identification.

Par ailleurs, l'obscénité peut être au cœur du message de l'artiste qui témoigne de l'effraction que produisent ces techniques. Retratos intimos (2003) de Cris Bierrenbach est une série de cinq agrandissements de photographies numériques de radiographies qui montrent l'intérieur du corps, de la hauteur de l'estomac aux genoux, avec cinq objets coupants et pointus (seringue, fourchette, ciseaux, couteau, forceps) mêlés à de la vaseline et insérés dans un vagin.

Le radiologue et plasticien, Rodolphe von Gombergh, développe une technique d'imagerie médicale qui permet d'examiner l'intérieur du corps avec une grande précision sans intrusion d'une caméra dans l'organisme. Dans le domaine médical, ces images en 3D sont précieuses pour les médecins comme pour les patients puisqu'elles évitent toute anesthésie ou hospitalisation tout en permettant de voyager dans les moindres recoins du corps humain. Sur le champ artistique, ces images spectaculaires invitent à un voyage entre réalité et fiction. Avec I'affichage du corps en relief, un chirurgien peut opérer son patient à distance, grâce à un robotmanipulateur, avec une meilleure précision. En conservant la sensation du volume et des distances entre les organes. II estime que l'affichage des clichés des poumons en 3D pourrait faciliter la détection d'une anomalie. Étrange alliance entre l'art et la science, comme mise en scène des Femmes transparentes, qui par l'incandescence qu'elles recèlent, s'inaugurent comme fétiche.

En définitive, ce que nous voyons est inséparablement lié à et dépend de notre manière de le voir. L'enjeu de mon travail se précise ici dans l'idée selon laquelle, face à l'effet engagé dans l'épreuve spéculaire, l'écoute clinique des malades qui y sont confrontés, comme de tout un chacun influé par la médiatisation des icônes médicales, a pour enjeu de permettre au sujet de se faire un regard. Regard confisqué par le discours technoscientifique en tant que tel.

\section{Conclusion}

Ce que nous voyons dépend de notre manière de le voir. L'enjeu de mon travail se précise ici dans l'idée selon laquelle, face à la problématique identitaire engagée dans l'épreuve spéculaire, l'écoute clinique des malades qui y sont confrontés, comme de tout sujet contemporain dans son rapport à la médecine, a pour enjeu de permettre au sujet de se faire un regard. Regard confisqué par le discours technoscientifique en tant que tel.

Pour le clinicien, l'enjeu est d'accompagner le processus de changement, il ne s'agit pas de se 
montrer prospectiviste. La technique, aujourd'hui, engage des modifications importantes dans l'appréhension du réel et du monde. Cette spécificité est à penser tant du point de vue du normal que du pathologique.

Leroi Ghouran a bien montré comment les outils techniques participent du processus d'hominisation. Chaque époque incorpore son progrès, change l'homme en le rendant dépendant des prothèses qui lui ouvrent des possibles. Cette donnée anthropologique est confirmée par le psychanalyste Harold Searle. Dans L'environnement non humain, il fait remarquer que l'élément non humain de l'environnement de I'homme forme l'un des constituants les plus fondamentaux de la vie psychique.
Pour Searle, la capacité ou l'incapacité de I'humain à entretenir avec son entourage non humain une relation constructive, contribue à son équilibre ou son déséquilibre psychique.

La technique n'est donc pas neutre.

L'art invite à libérer la pensée associative face à ces images du corps, à en interroger la dimension psychique et politique. Elle interroge ce rôle par rapport à l'identité. La psychanalyse permet l'énonciation des enjeux psychiques liés à cette expérience. Cette illustration par l'art de la représentation du corps résonne dans les paroles entendues au sein de dispositifs d'écoute à l'hôpital, de groupes de paroles ; dans tous ces lieux d'échanges, à la croisée des regards.

\section{Bibliographie :}

Agamben, G. (2007). Qu'est-ce qu'un dispositif ? Paris : Éd. Payot \& Rivages.

Ferenczi, S. (1933). L'influence de Freud sur la médecine.

Foucault, M. (2001). Dits et écrits, 1954-1988. Paris :

Gallimard.

Horio Monteiro, R. (2007). Images médicales entre art et science. Sociétés, 95.

Lacan, J. (1966). La place de la psychanalyse dans la médecine. Conférence. Le Bloc-Notes de la psychanalyse, 7 (9-40), Georg éditeur.

Lacan, J. (1966). Écrits. Paris : Seuil.

Lacan, J. (1992). Les Quatre concepts fondamentaux de la psychanalyse (1964). Le Séminaire, XI. Paris : Seuil.

Potier, R. (2007). L'imagerie médicale à l'épreuve du regard : enjeux éthiques de l'expérience face à l'image. Cliniques méditerranéennes: Psychanalyse et Psychopathologie Freudiennes. 76, 2007, 77-90.

Potier, R. (2009). L'image en médecine, esquisse et précipice. Recherches en Psychanalyse, 8|2009

Slatman, J. (2004). L'imagerie du corps interne. Penser le corps.

\section{Notes :}

${ }^{1}$ Transference : " Le transfert, en psychanalyse, est essentiellement le déplacement d'une conduite émotionnelle par rapport à un objet infantile, spécialement les parents, à un autre objet ou à une autre personne, spécialement le psychanalyste au cours du traitement. » (Daniel Lagache, 1949, p. 33)
${ }^{2}$ La vidéo est projetée de manière circulaire sur le sol d'une structure cylindrique dans laquelle le spectateur pénètre par l'une des deux portes étroites situées de part et d'autre. Une bande sonore, diffusant les pulsations cardiaques telles qu'on les entend depuis les différentes parties du corps examinées par la caméra, accompagne l'image vidéo. Durant une dizaine de minutes, la caméra, privilégiant les mouvements de va-et-vient, parcourt à un rythme rapide les contours du corps de l'artiste, s'introduisant successivement dans ses divers orifices.

${ }^{3}$ Slatman, Jenny (2004). L'imagerie du corps interne. Penser le corps, 4.

${ }^{4}$ Ibid.

${ }^{5}$ Horio Monteiro, Rosana (2007/1). Images médicales entre art et science. Sociétés, $\mathrm{n}^{\circ} 95$.

${ }^{6}$ Rosana Horio Monteiro témoigne ainsi du travail de l'artiste : "La désindividualisation du sujet contemporain est un autre élément du travail de l'artiste. C'est le vestige sans nom de l'examen médical. " "Le regard n'identifie pas ; l'œil dit seulement que cela est un corps humain ", affirme Monica Mansur. "Si c'est un homme ou une femme, vieux ou jeune, il n'est pas possible de le savoir sans la connaissance médicale spécialisée ; ce sont des visions médicales qui existent seulement parce qu'elles ont été "imaginées" à travers une machine, soit une caméra vidéo avec fibre optique, un tunnel avec des ondes magnétiques ou des rayons lasers qui plaquent des coupes transversales d'organes et os ", complète l'artiste Rosana Horio Monteiro, Ibid. 


\section{L'auteur :}

\section{Rémy Potier}

Docteur en Psychopathologie / Phd. Maître de Conférences en Psychopathologie. Centre de Recherches Psychanalyse, Médecine et Sociétés - EAD N³522. Université Paris VII Diderot.

Campus Paris Rive Gauche

Bâtiment Olympe de Gouges

11, rue Jean Antoine de Baïf

75013 Paris

France

\section{Référence électronique}

Rémy Potier, " Imagerie médicale et art contemporain, rencontres autour d'un corps virtuel ", Recherches en Psychanalyse [En ligne], 12 |2011, mis en ligne le 22 décembre 2011.

Texte intégral

Droits d'auteur

Tous droits réservés 